\title{
Learning curves of basic laparoscopic psychomotor skills in SINERGIA VR simulator
}

\author{
L. F. Sánchez-Peralta - F. M. Sánchez-Margallo • \\ J. L. Moyano-Cuevas · J. B. Pagador - S. Enciso · \\ E. J. Gómez-Aguilera · J. Usón-Gargallo
}

\begin{abstract}
Purpose Surgical simulators are currently essential within any laparoscopic training program because they provide a low-stakes, reproducible and reliable environment to acquire basic skills. The purpose of this study is to determine the training learning curve based on different metrics corresponding to five tasks included in SINERGIA laparoscopic virtual reality simulator.

Methods Thirty medical students without surgical experience participated in the study. Five tasks of SINERGIA were included: Coordination, Navigation, Navigation and touch, Accurate grasping and Coordinated pulling. Each participant was trained in SINERGIA. This training consisted of eight sessions (R1-R8) of the five mentioned tasks and was carried out in two consecutive days with four sessions per day. A statistical analysis was made, and the results of R1, R4 and R8 were pair-wise compared with Wilcoxon signed-rank test. Significance is considered at $P$ value $<0.005$.

Results In total, $84.38 \%$ of the metrics provided by SINERGIA and included in this study show significant differences when comparing R1 and R8. Metrics are mostly improved in the first session of training $(75.00 \%$ when $\mathrm{R} 1$ and $\mathrm{R} 4$ are compared vs. $37.50 \%$ when $\mathrm{R} 4$ and $\mathrm{R} 8$ are compared). In tasks Coordination and Navigation and touch, all metrics
\end{abstract}

are improved. On the other hand, Navigation just improves $60 \%$ of the analyzed metrics. Most learning curves show an improvement with better results in the fulfillment of the different tasks.

Conclusions Learning curves of metrics that assess the basic psychomotor laparoscopic skills acquired in SINERGIA virtual reality simulator show a faster learning rate during the first part of the training. Nevertheless, eight repetitions of the tasks are not enough to acquire all psychomotor skills that can be trained in SINERGIA. Therefore, and based on these results together with previous works, SINERGIA could be used as training tool with a properly designed training program.

Keywords Laparoscopy · Simulator · Training · Virtual reality $\cdot$ Validation $\cdot$ Learning curve

\section{Introduction}

Laparoscopy has become a gold standard in many surgical procedures for the last years. This fact is mainly thanks to its application in different surgical procedures and its multiple benefits for patients as well as for health systems [1-4].

These new laparoscopic techniques imply the acquisition of laparoscopic psychomotor skills in addition to the typical surgical skills [5] principally due to the following differences, among others, when compared with open surgery [6]:

- The laparoscope changes the 3D surgical field into a 2D view of it on the screen.

- This conversion results on a loss of depth perception.

- There is not a direct tactile perception but through a long, thin and without "wrists" surgical instrument.

- The fulcrum effect, due to the use these instruments through a fixed point, results in non-intuitive movements. 
So, it is highly important to provide opportunities for surgical skills training and to find adequate time for trainees to practice and master their skills. This teaching and practice should occur outside the operating room [7] due to issues such as quality control, patient safety, financial constraints, efficiency and cost-effectiveness and less exposure [8]. Thanks to training, some behaviors, skills and surgical gestures can be made automatically so the limited attentional resources that should be dedicated to them can be focused on other activities like decision making [9].

Simulators are becoming an important method for surgical training because they offer a low-stakes, learner-centered education as well as a reliable and reproducible environment [10-13] and there is a wide literature proving its utility [1418]. Simulators are usually classified in three categories: box trainers, augmented reality simulators and virtual reality simulators. Virtual reality (VR) simulators objectively assess the performance of the tasks without requesting the intervention of an expert since they measure and store metrics during the performance itself $[19,20]$.

A VR simulator has to be reliable and valid if we want to use it as training tool. Usually there are five types of validations: face validity, content validity, construct validity, concurrent validity and predictive validity $[6,20,21]$ but besides that, it is also interesting to analyze the learning curve of trainees in order to see the evolution of metrics along the repetitions. The learning curve shows the number of times that a particular task or procedure must be done in order to complete it repeatedly with high accuracy and precision [22].

SINERGIA [23] is a VR simulator for training basic skills in laparoscopic surgery. Although different validation studies have been performed [24,25], the learning curve of novices has not been analyzed yet. Therefore, the objective of this work is to determine and analyze this learning curve for different metrics corresponding to five tasks included in SINERGIA VR simulator.

\section{Materials and methods}

Subjects

Thirty participants were recruited for the study. They were medical students ( 21 women and 9 men) who had few or no experience in laparoscopic surgery neither had trained in any virtual laparoscopic surgical simulator. Mean age of the participant was $24.3 \pm 4.3$ years (range, $20-38$ years). Twentysix participants in the study were right-handed, 2 participants were left-handed and 2 participants were ambidextrous. All tests were accomplished in the Jesús Usón Minimally Invasive Surgery Centre in Cáceres, Spain.

\section{Training in SINERGIA}

SINERGIA [23] is a VR simulator designed for the learning of basic skills in laparoscopic surgery. Two main contributions arise from this global aim: (1) a specification of didactic exercises that has reverted into the creation of a new training environment; (2) an appropriate use of the simulations technologies that significantly improved the whole simulation. The simulator comprises 7 didactic units: hand-eye coordination, camera manipulation, grasping, pulling, cutting, dissection and suture; with one or more tasks in each one of them.

Among all the tasks SINERGIA provides, five tasks have been selected for this study (Fig. 1) and the rest have been omitted due to subject time considerations. For each of these tasks, a certain number of metrics have been automatically recorded. Some parameters are common between tasks whereas other metrics are specific to certain tasks. Table 1 shows a definition of the tasks as well as which metrics are recorded for each one. A definition of the metrics is provided in [24]. Common metrics are total time, fulfillment and instrumental efficiency. Path length is also recorded by the

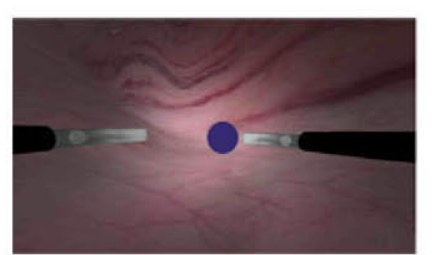

Coordination

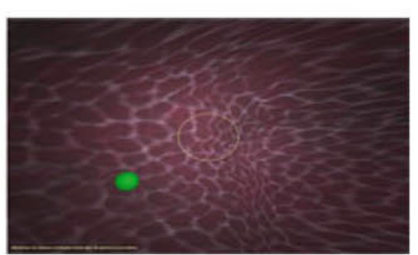

Navigation

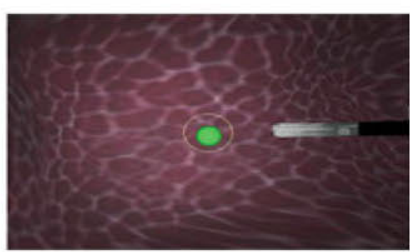

Navigation and touch

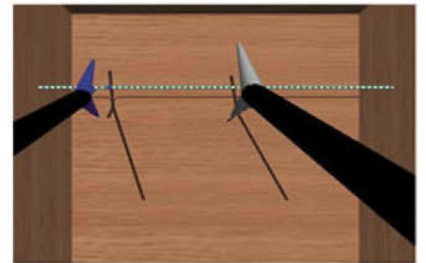

Accurate grasping

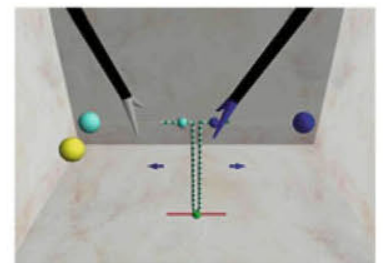

Coordinated pulling

Fig. 1 SINERGIA tasks included in this study 
Table 1 Automatically evaluated metrics in SINERGIA divided by tasks

\begin{tabular}{|c|c|c|}
\hline Task & Description & Metrics \\
\hline Coordination & $\begin{array}{l}\text { Touching static spheres that } \\
\text { appear sequentially in an } \\
\text { "organic scene". There is a } \\
\text { time limit to touch each } \\
\text { sphere }\end{array}$ & $\begin{array}{l}\text { Total time }(\mathrm{s}) \\
\text { Partial time }(\mathrm{s}) \\
\text { Fulfillment }(\%) \\
\text { Left instrument efficiency }(\%) \\
\text { Right instrument efficiency }(\%) \\
\text { Harm to background }(\#)\end{array}$ \\
\hline Navigation & $\begin{array}{l}\text { Centering an endoscope sight } \\
\text { in spheres that sequentially } \\
\text { appear. There is a time limit } \\
\text { to center each sphere }\end{array}$ & $\begin{array}{l}\text { Total time }(\mathrm{s}) \\
\text { Partial time }(\mathrm{s}) \\
\text { Fulfillment }(\%) \\
\text { Left instrument efficiency }(\%) \\
\text { Harm to background (\#) }\end{array}$ \\
\hline Navigation and touch & $\begin{array}{l}\text { Centering an endoscope sight } \\
\text { in a sphere and once it is } \\
\text { centered, touching it with the } \\
\text { other tool. Spheres appear } \\
\text { sequentially. There is a time } \\
\text { limit to center and touch each } \\
\text { sphere }\end{array}$ & $\begin{array}{l}\text { Total time (s) } \\
\text { Partial time (s) } \\
\text { Fulfillment }(\%) \\
\text { Left instrument efficiency }(\%) \\
\text { Harm to background (\#) }\end{array}$ \\
\hline Accurate grasping & $\begin{array}{l}\text { Grasping certain points of a } \\
\text { thread without causing } \\
\text { deformations to it. Grasping } \\
\text { areas appear sequentially. } \\
\text { There is a time limit to grasp } \\
\text { the point }\end{array}$ & $\begin{array}{l}\text { Total time (s) } \\
\text { Partial time }(\mathrm{s}) \\
\text { Fulfillment }(\%) \\
\text { Deviation from the center point }(\mathrm{cm}) \\
\text { Left instrument efficiency }(\%) \\
\text { Right instrument efficiency }(\%) \\
\text { Grasping out of the area }(\#) \\
\text { Grasping with excessive pressure (\#) }\end{array}$ \\
\hline Coordinated pulling & $\begin{array}{l}\text { Grasping a thread at the marked } \\
\text { points and pull them following } \\
\text { the white path until the big } \\
\text { spheres. A "coordination-control } \\
\text { bar" provides formative feedback }\end{array}$ & $\begin{array}{l}\text { Total time (s) } \\
\text { Partial time (s) } \\
\text { Fulfillment }(\%) \\
\text { Left instrument distance to the ideal } \\
\text { path }(\mathrm{cm}) \\
\text { Right instrument distance to the } \\
\text { ideal path (cm) } \\
\text { Left instrument efficiency (\%) } \\
\text { Right instrument efficiency (\%) } \\
\text { Non-coordination moments (\#) }\end{array}$ \\
\hline
\end{tabular}

simulator but it has not been included in the study. Although this metric is quite intuitive, it is not useful to compare as it is highly dependent on the position of the elements which are randomly situated on the scene. Therefore, efficiency is more recommended as the effects of the random positions are avoided.

\section{Study design}

All participants were trained in SINERGIA. The study methodology is shown in Fig. 2. The training consisted of 8 repetitions with 5 tasks each and was made in two sessions in consecutive days with 4 repetitions per day.

An instructor was available at all sessions, providing standardized oral feedback about tasks and rules before the first run of each task. Subjects were supervised while they were accomplishing the tasks but they did not receive any external help by the instructor.

Each repetition lasted 10 min except for the first one because of the explanations provided by the instructor to the participant. As time to accomplish each task is short, no break between tasks was included (just the necessary time to change between tasks in SINERGIA VR simulator). Break between repetitions was approximately 2 min long.

\section{Statistical analysis}

Statistical analysis was performed using SPSS software (Version 15.0, SPSS Inc, Chicago, IL, USA). Data are given as mean \pm standard deviation (SD). Learning curves have been graphically represented, considering results from $\mathrm{R} 1$ to $\mathrm{R} 8$. 


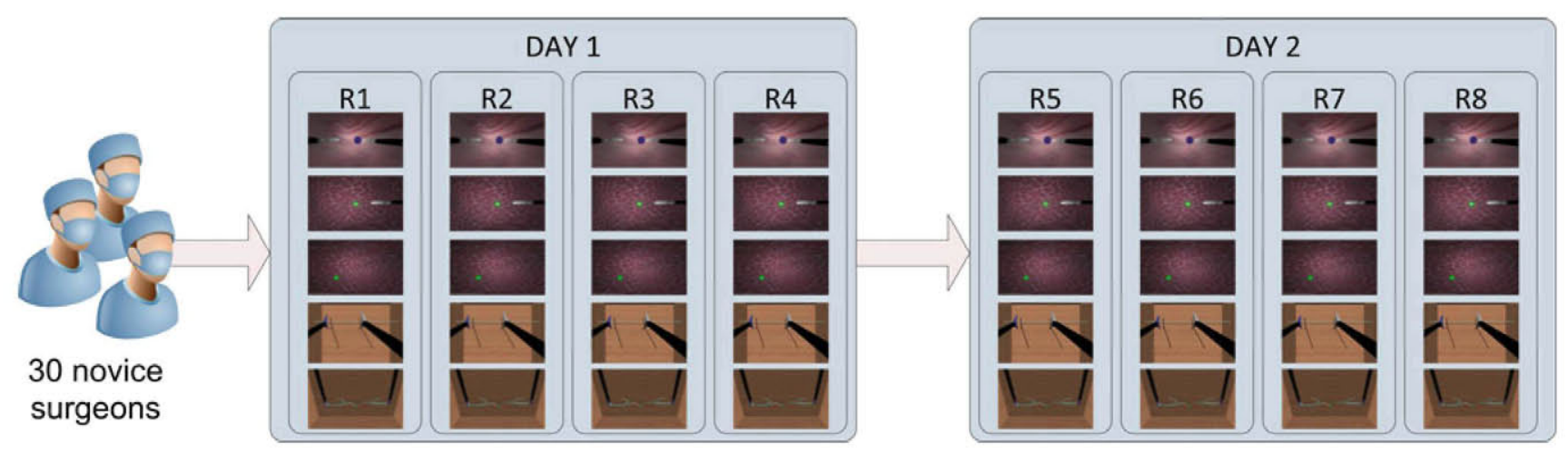

Fig. 2 Study methodology. All participants (30 novice surgeons) performed eight iterations (R1-R8) consisting in 5 selected tasks of SINERGIA VR simulator. This training was carried out in two consecutive days

Wilcoxon signed-rank test was used to analyze the differences between different stages of the training:

- The first and the last repetitions of the first training session, R1 and R4, respectively,

- the last repetition of each training session, R4 and R8, and

- the first and the last repetitions of the training session, R1 and $\mathrm{R} 8$, respectively.

Differences were considered significant at $P<0.05$.

\section{Results}

Table 2 shows the results of R1, R4 and R8. All metrics for each task have been included in the table. For the five selected tasks, novices significantly improve their skills in most evaluated metrics. Furthermore, despite some metrics do not present significant differences between $\mathrm{R} 1$ and $\mathrm{R} 8$, the obtained mean values are in all cases improved except in the case of deviation of the central point in task Accurate Grasping, where mean value remains approximately constant.

In general terms, most metrics that presents significant differences when comparing R1 and R8 (84.38\%) are already significant when comparing first and last repetition of the first training session (R1 vs. R4; significance in $75.00 \%$ of the metrics). Differences are lower in the second part of the training, finding fewer cases of significant differences in the comparison of R4 and R8 (significance in $37.50 \%$ of the metrics). The exception to this situation is found in Navigation and touch, where there are more cases of significant differences when comparing R4 and R8 than when comparing R1 and R4.

As described in the methodology section, metrics that are evaluated in all tasks are total time, fulfillment and left instrument efficiency. Right instrument efficiency is also measured in all tasks except in tasks where camera handling is involved.
Figures $3,4,5,6$ show the mean value of these four metrics. Error bars represent a $95 \%$ confidence interval. Some points in the figures are slightly displaced in order to avoid error bars to overlap. Total time shows a decreasing trend in all cases, most marked in Coordinated pulling. Fulfillment learning curve presents the most pronounced improvement along repetitions, especially coordinated pulling where it increases from $65.56 \pm 28.34$ in R1 to $96.77 \pm 10.01$ in R4. Left instrument efficiency and right instrument efficiency show in both cases an increasing behavior but the improvement rate is lower than other metrics.

\section{Discussion}

Laparoscopic surgery implies the acquisition of new surgical skills that are very different to the ones of open surgery and besides that, there is not transference of skills between the two types of surgery $[6,26,27]$. Learning curve of laparoscopic surgery appears to be longer than the one of open surgery $[6,22]$ so surgical simulators have been used to shorten this learning curve by acquiring basic skills out of the operating room and automating them [9].

Therefore, this paper presents the learning curve of novices when they train basic laparoscopic psychomotor skills in SINERGIA VR laparoscopic simulator. By itself, this type of curves does not necessarily imply a learning of skills but it is often considered an interesting step in the global evaluation of a simulator since it warrants further investigation and potentially can lead to a generalization of the skills acquisition [12]. The motivation to carry out this study is based on the fact that SINERGIA virtual reality simulator is currently a prototype so all different approaches regarding the full validation of the training system must be addressed. Therefore, our final aim is to check a new virtual reality simulator that allows training basic skills in a safe, reliable and reproducible environment. 


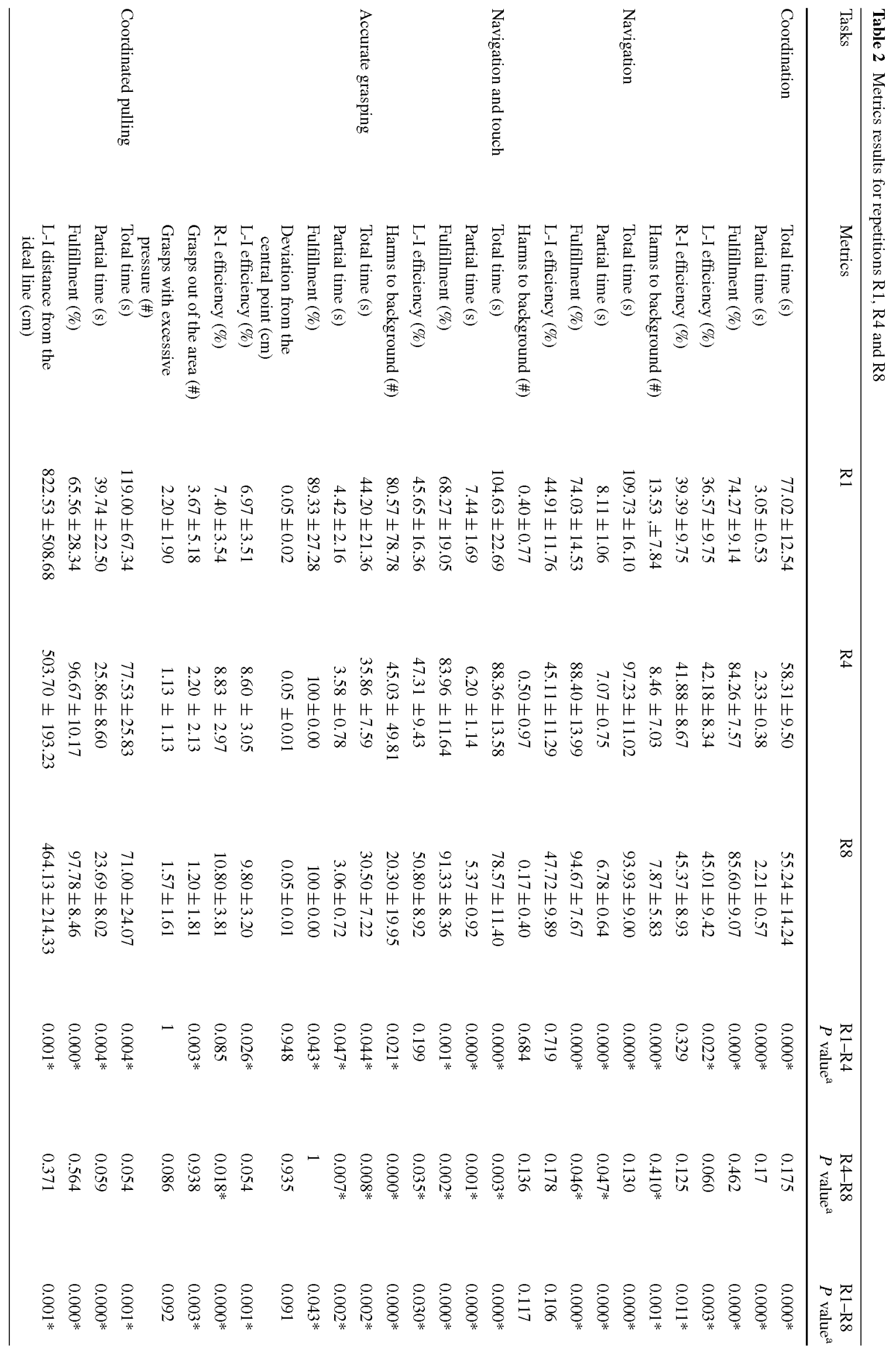




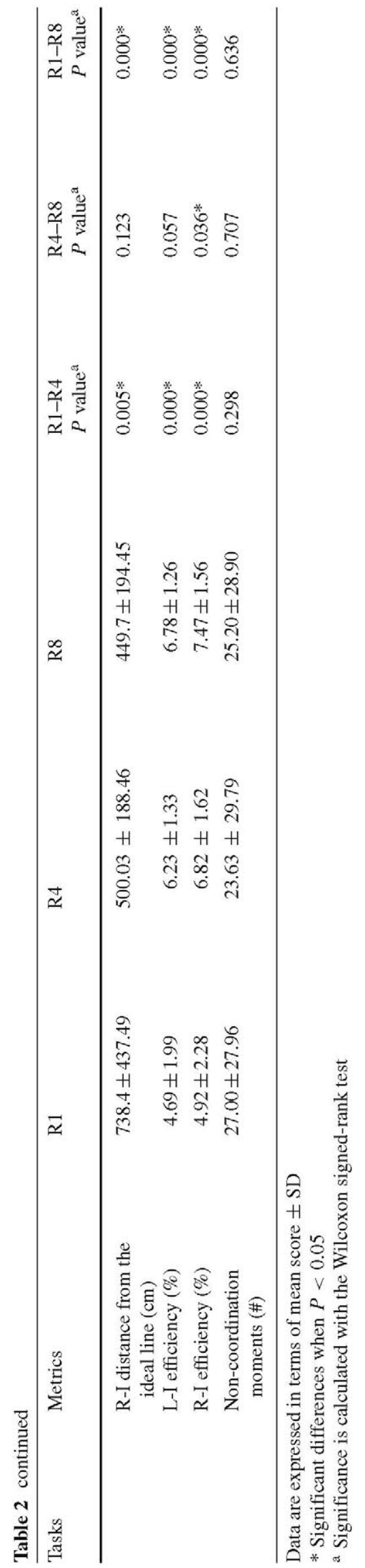

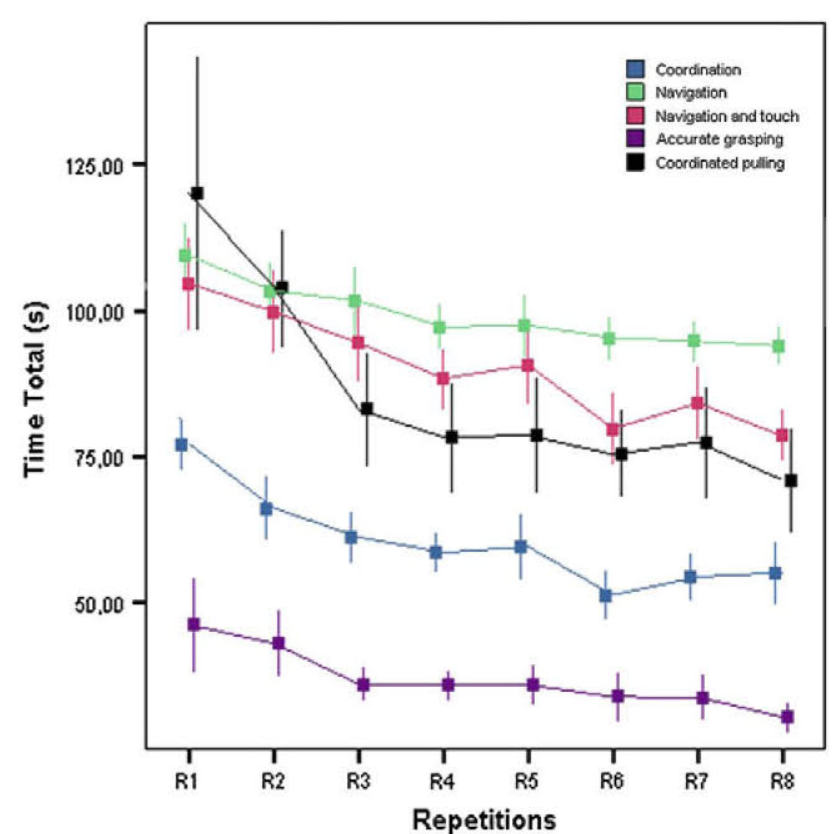

Fig. 3 Learning curves corresponding to total time to finish the task (s). Error bars represent a $95 \%$ confidence interval

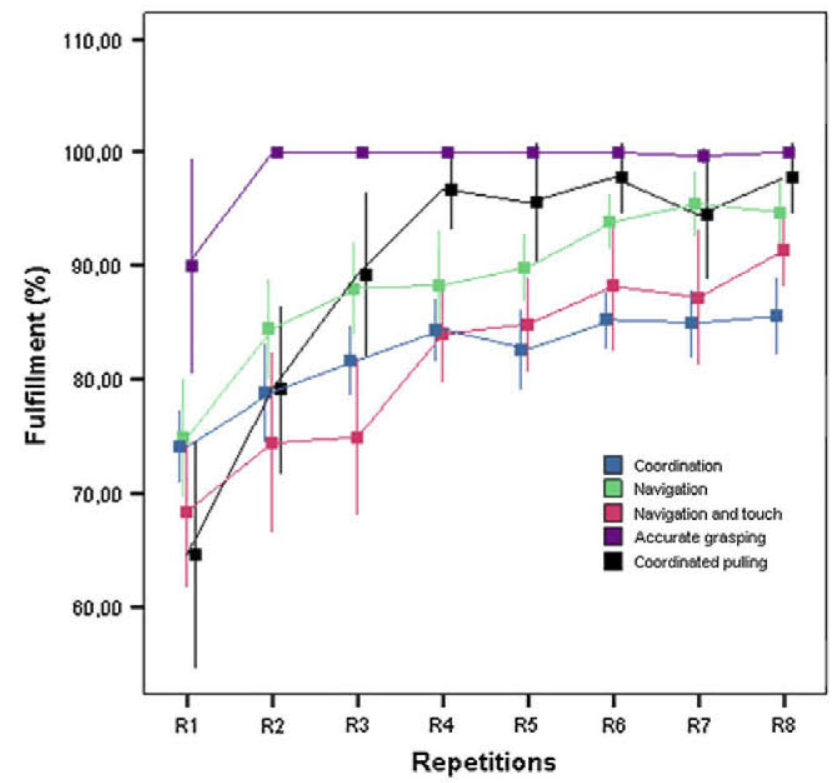

Fig. 4 Learning curves corresponding to fulfillment (\%). Error bars represent a $95 \%$ confidence interval

The defined training program included eight repetitions of the selected tasks, divided in two sessions in consecutive days. The determination of choosing eight and no more repetitions was based on the results of Grantcharov et al. [28]. They exposed that learning curves for novice surgeons reached plateau after seven repetitions of the task in a VR simulator. Besides that, we have observed in our study that although learning curves corresponding to half of the analyzed metrics reach plateau in the fourth iteration, there are still other metrics that after eight sessions could be improved 


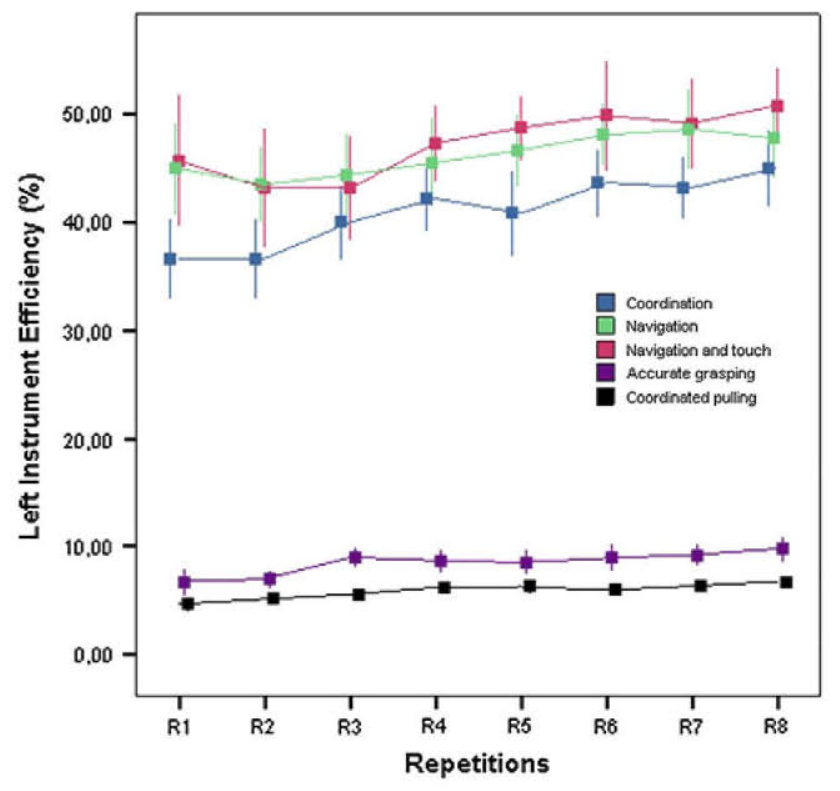

Fig. 5 Learning curves corresponding to left instrument efficiency (\%). Error bars represent a $95 \%$ confidence interval

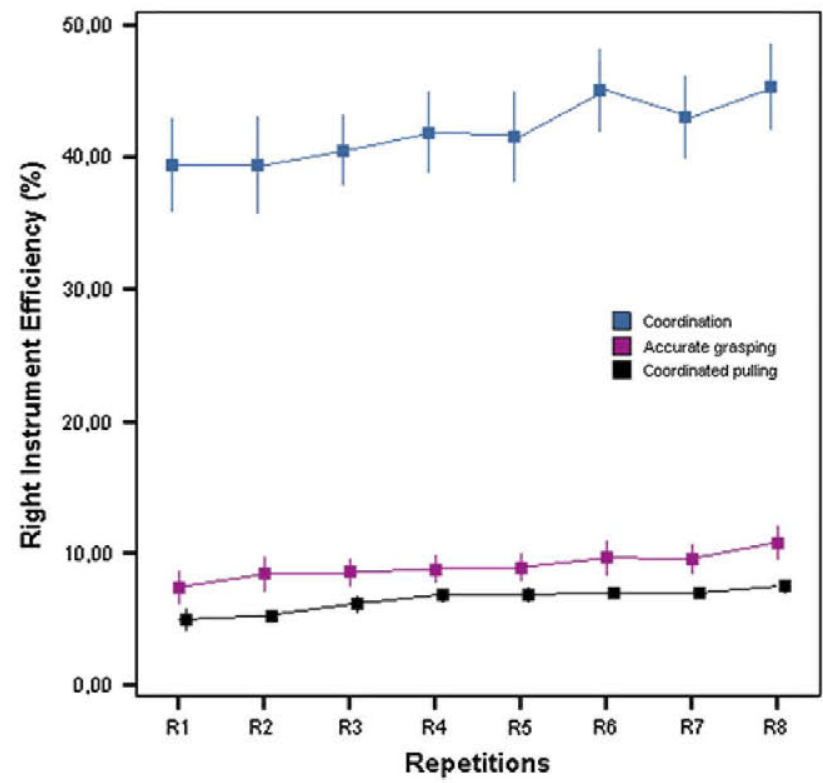

Fig. 6 Learning curves corresponding to right instrument efficiency (\%). Error bars represent a $95 \%$ confidence interval

with a longer training program. This may be due to a different level of difficulty of the tasks considered or to a different training program. On the other hand and in our opinion, a possible 'overtraining' in basic skills would never be damaging but on the contrary it could be used as warming up before performing more difficult training tasks or even a real procedure. This has been already addressed by Plerhoples et al. in [29] where they prove that using a mobile device balance game decreases errors on basic tasks performed on a laparoscopy surgery simulator.
However, the optimum training program has not been defined yet [30] and the structure of that practice has to be determined too because only a lengthy enough practice is not sufficient [13]. Although surgeons normally acquire surgical skills in short courses [30], it seems that distributed training is superior to massed training in acquiring and retaining psychomotor skills for endoscopic surgery on a VR simulator [31,32]. In our case, it was not possible to carry out a distributed program due to subject time constraints, but we consider that results obtained in our study could be also improved with a distributed training program.

After an 8-repetition training program for basic surgical psychomotor skills training in SINERGIA, novices' performances were significantly better in most metrics for the analyzed tasks. Coordination and Navigation and touch improve all metrics along the whole training program. On the other hand, Navigation just improves in $60 \%$ of them.

In Coordination task, metrics are mainly improved during the first training session. Hand-eye coordination skill, although not specifically, is also trained when the other tasks are accomplished. This could explain the fact that total time or harms to background are mainly improved from R1 to R4 and differences are not significant when comparing R4 and R8. On the other hand, efficiency, both for the left and the right instruments, as well as Harms to the background are improved along the 8 repetitions and in our opinion they could be still improved with more training sessions. Comparing these results with the mean of experts obtained in [24], novices are not able to reach experts' level just with an 8 -repetition training program. Trainees acquire confidence to handle instruments and move them faster, but they still are not able to move them efficiently without damaging the background.

Metrics of Navigation are improved during the whole training program in most of the cases. On the other hand, this task presents significant differences in a lower number of metrics. Trainees are able to finish more subtasks as the training goes on, as it is shown by the reduction of Total time and the increment of Fulfillment, but more training would be necessary to reduce the path length of the camera to focus the spheres without damaging the anatomical structures in the surgical scene. This is consistent with the results exposed by Moyano-Cuevas et al. [25], which shows that training in SINERGIA allows improving skills regarding the precision, finding and focusing of structures. Since they did not measure efficiency or harms with LapMentor, those aspects could not be detected in their study.

As mentioned in the results section, Navigation and touch is the only task that presents more significant differences when the second part of the training is analyzed. It is left instrument efficiency the metric that does not improve significantly from R1 to R4. This may be due to this task requires a different maneuver for each hand (the sphere must be focused 
with the left instrument and touched with the right one) and just four iterations are not enough for novices to improve the accomplishment of two different actions at the same time in an effective way. Nevertheless, novices improve their efficiency in the second part of the training but we consider that better results can be achieved with further training since their performance is still distant to results of experts showed in [24].

Task Accurate grasping presents the fastest acquisition rate of all considered tasks. It is interesting to remark results corresponding to the metric Grasps with excessive pressure. Although novices improve with training, differences are not significant. This is probably due to the fact that SINERGIA does not present a good realistic feedback as haptic sensation was rated with a low punctuation in the face validity of the simulator [24]. Besides that, experts achieve a perfect score in this metric $(0.00 \pm 0.00$ errors, as shown in [24]). With these results, it can be concluded that SINERGIA is not the best environment to acquire tactile sensation and handling of tissues but it is able to distinguish when too much pressure is done.

Metrics corresponding to Coordinated pulling task show that novices easily improve time and fulfilment. On the contrary, 3D perception can still be improved since they move instruments far away from the ideal path. Furthermore, noncoordination moments do not get close to the level of experts that barely reaches a mean value equal to 1.5 moments [24].

It is interesting to remark the efficiency values in the case of Accurate grasping and Coordinated pulling. Efficiency is defined in [24] as the ratio between the minimum distance and the actual distance to accomplish the task. Particularly for Accurate grasping, these low values are due to the fact that minimum distance to accomplish the task is the straight line between the point to be grasped and the tool tip, which is in some cases, when two consecutive points have to be grasped with the same hand, the distance between the points along the thread. This is the minimum distance but in any case it is the natural way of behavior since surgeons tend to make a retraction movement to approach again the thread instead of moving the tool tip along the thread. This retraction movement makes that the actual distance is much greater than the minimum distance, resulting in low values of efficiency. The suitability of this minimum distance as optimal path in MIS has been already studied by Chmarra et al. [33] who present the concepts of retracting and seeking movements, concluding that the shortest path length, as presently used during the assessment of basic MIS skills, may be not a proper concept for analyzing optimal movements and therefore needs to be revised.

In general terms, novices accomplish all tasks in less time but that does not imply an improvement in the efficiency of movements. Therefore, the more the novices train, the faster they move the instrument but they do not do it in the most cor- rect way. A similar result can be found in [34], where Pagador et al. expose that intermediate surgeons could feel self-confident and move tools quicker than needed when accomplishing a surgeon's knot. We agree with Pagador et al, because in our opinion, repeating a task without proper feedback or mentoring do not lead to an expert execution which is also supported by Kolozsvari et al. in [13] where they state the four criteria requested for a deliberate practice: improve a specific aspect of performance; the need of valid, thorough and immediate feedback; opportunity to perform the tasks repeatedly within a controlled environment; and training sessions limited to $1 \mathrm{~h}$. Therefore, VR simulators as SINERGIA together with a suitable mentorship could be a proper environment for this deliberate practice.

In light of the results of the study, learning curves of metrics that assess the basic psychomotor laparoscopic skills acquired in SINERGIA VR simulator show a faster learning rate during the first part of the training. Nevertheless, eight repetitions of the tasks are not enough to acquire all psychomotor skills that can be trained in SINERGIA. Therefore, with a properly designed training program, SINERGIA could be used as training tool. In our opinion, training programs must be distributed rather than massed in order to increase retention of psychomotor skills but they also have to take into account time constraints due to the maximum time that trainees can be out of their working place. Therefore, a proper training program would include an in-person training stage with a short duration that would be complemented with a distance training stage that would allow the trainee to train from his/her working place or even from home. VR simulators such as SINERGIA could be used in any of these two stages, although the usually high cost of this type of training devices limits a wider use.

Brinkman et al. [35] concluded that criterion-based training of laparoscopic skills can reduce the overall training time with no impact on training outcome, transferability or retention of skills. Therefore, it is considered as future work whether it is possible to improve the learning curve shown in this paper by establishing as benchmark the results of experts and determine whether results of Brinkman et al. are also transferrable to a VR simulator.

Acknowledgments This work has been funded by SINERGIA Cooperative Network (G03/135) from the Ministerio de Sanidad y Consumo, Spain.

Conflict of interest None.

\section{References}

1. Wang MY, Cummock MD, Yu Y, Trivedi RA (2010) An analysis of the differences in the acute hospitalization charges following minimally invasive versus open posterior lumbar interbody fusion. J Neurosurg Spine 12(6):694-699 
2. Da Luz Moreira A, Kiran RP, Kirat HT, Remzi FH, Geisler DP, Church JM et al (2010) Laparoscopic versus open colectomy for patients with American Society of Anesthesiology (ASA) classifications 3 and 4: the minimally invasive approach is associated with significantly quicker recovery and reduced costs. Surg Endose 24(6):1280-1286

3. Lazzarino AI, Nagpal K, Bottle A, Faiz O, Moorthy K, Aylin $P$ (2010) Open versus minimally invasive esophagectomy: trends of utilization and associated outcomes in England. Ann Surg 252(2):292-298

4. Wei B, Qi CL, Chen TF, Zheng ZH, Huang JL, Hu BG, Wei HB (2011) Laparoscopic versus open appendectomy for acute appendicitis: a metaanalysis. Surg Endosc 25(4):1199-1208

5. Molinas CR, De Win G, Ritter O, Keckstein J, Miserez M, Campo R (2008) Feasibility and construct validity of a novel laparoscopic skills testing and training model. Gynecol Surg 5(4):281-290

6. Fowler DL (2010) Enabling, implementing and validating training methods in laparoscopic surgery. World J Surg 34(4):621-624

7. Goff BA (2010) Training and assessment in gynaecologic surgery: the role of simulation. Best Pract Res Clin Obstet Gynaecol 24(6):759-766

8. Kolkman W, van de Put MAJ, Wolterbeek R, Trimbos JBMZ, Jansen FW (2008) Laparoscopic skills simulator: construct validity and stablishment of performance standards for residency training. Gynecol Surg 5:109-114

9. Gallagher AG, Ritter EM, Champion H, Higgins G, Fried MP, Moses G et al (2005) Virtual reality simulation for the operating room. Proficiency-based training as a paradigm shift in surgical skills training. Ann Surg 241(2):364-372

10. Fried GM, Feldman LS, Vassiliou MC, Fraser SA, Stanbridge D, Ghitulescu G et al (2004) Proving the value of simulation in laparoscopic surgery. Ann Surg 240(3):518-528

11. Usón J, Sánchez FM, Sánchez MA, Pérez FJ, Hashizume M (2007) Simuladores Laparoscópicos. In: Uson J, Sánchez FM, Pascual S, Climent S (eds) Formación en Cirugía

12. Kahol K, Vankipuram M, Smith ML (2009) Cognitive simulators for medical education and training. J Biomed Inf 42(4): 593-604

13. Kolozsvari NO, Feldman LS, Vassiliou MC, Demnyttenaere S, Hoover ML (2011) Sim one, do one, teach one: considerations in designing training curricula for surgical simulation. J Surg Educ 68(5):421-427

14. Kanumuri P, Ganai S, Wohaibi EM, Bush RW, Grow DR, Seymour NE (2008) Virtual reality and computer-enhanced training devices equally improve laparoscopic surgical skill in novices. JSLS 12(3):219-226

15. Gauger PG, Hauge LS, Andreatta PB, Hamstra SJ, Hillard ML, Arble EP et al (2010) Laparoscopic simulation training with proficiency targets improves practice and performance of novice surgeons. Am J Surg 199(1):72-80

16. Sroka G, Feldman LS, Vassiliou MC, Kaneva PA, Fayez R, Fried GM (2010) Fundamentals of laparoscopic surgery simulator training to proficiency improves laparoscopic performance in the operating room-a randomized trial. Am J Surg 199(1): $115-120$

17. Sturm LP, Windsor JA, Cosman PH, Cregan P, Hewett PJ, Maddern GJ (2008) A systematic review of skills transfer after surgical simulation training. Ann Surg 248:166-179

18. Stelzer MK, Abdel MP, Sloan MP, Gould JC (2009) Dry lab practice leads to improved laparoscopic performance in the operating room. J Surg Res 154:163-166
19. Botden SM, Jakimowicz JJ (2009) What is going on in augmented reality simulation in laparoscopic surgery. Surg Endosc 23(8):1693-1700

20. Thijssen AS, Marlies PS (2010) Contemporary virtual reality laparoscopy simulators: quicksand or solid grounds for assessing surgical trainees. Am J Surg 199(4):529-541

21. McDougall EM (2007) Validation of surgical simulators. J Endourol 21(3):244-247

22. Teplitz CJ (1991) The learning curve deskbook: a reference guide to theory, calculations and applications. Quo-rum, NewYork, NY

23. Lamata P, Gómez E, Sánchez-Margallo F, López Ó, Monserrat C, García V et al (2007) Sinergia laparoscopic virtual reality simulador: didactic design and technical development. Comput Methods Programs Biomed 85(3):273-283

24. Sánchez-Peralta LF, Sánchez-Margallo FM, Moyano-Cuevas JL, Pagador JB, Enciso-Sanz S, Sánchez-González Pet al (2010) Construct and face validity of Sinergia laparoscopic virtual reality simulator. Int J Comput Assist Radiol Surg 5(4):307-315

25. Moyano-Cuevas JL, Sánchez-Margallo FM, Sánchez-Peralta LF, Pagador JB, Enciso S, Sánchez-González P, Gómez-Aguilera EJ, Usón-Gargallo J (2011) Validation of SINERGIA as training tool: a randomized study to test the transfer of acquired basic psychomotor skills to LapMentor. Int J Comput Assist Radiol Surg 6(6):839846

26. Subramonian K, DeSylva S, Bishai $P$, Thompson P, Muir G (2004) Acquiring surgical skills: a comparative study of open versus laparoscopic surgery. Eur Urol 45(3):346-351

27. Figert PL, Park AE, Witzke DB, Schwartz RW (2001) Transfer of training in acquiring laparoscopic skills. J Am Coll Surg 193(5):533-537

28. Grantcharov TP, Bardram L, Funch-Jensen P, Rosenberg J (2003) Learning cuves and impact of previous operative experience on performance on a virtual reality simulator to test laparoscopic surgical skills. Am J Surg 185(2):146-149

29. Plerhoples TA, Zak Y, Hernandez-Boussard T, Lau J (2011) Another use of mobile device: warm-up for laparoscopic surgery. J Surg Res 170:185-188

30. Wallace T, Birch DW (2007) A needs-assessment study for continuing professional development in advanced minimally invasive surgery. Am J Surg 193(5):593-595

31. van Dongen KW, Mitra PJ, Schijven MP, Broeders IAMJ (2011) Distributed versus massed training: efficiency of training psychomotor skills. Surg Tech Dev 1(1):40-42

32. Verdaasdonk EGG, Stassen LPS, van Wijk RPJ, Dankelman $\mathrm{J}$ (2007) The influence of different training schedules on the learning of psychomotor skills for endoscopic surgery. Surg Endosc 21:214-219

33. Chmarra MK, Jansen FW, Grimbergen CA, Dankelman J (2008) Retracting and seeking movements during laparoscopic goal-oriented movements. Is the shortest path length optimal?. Surg Endosc 22:943-949

34. Pagador JB, Sánchez-Margallo FM, Sánchez-Peralta LF, SánchezMargallo JA, Moyano-Cuevas JL, Enciso-Sanz S, Usón-Gargallo U, Moreno J (2012) Decomposition and analysis of laparoscopic suturing task using tool-motion analysis (TMA): improving the objective assessment. Int J Comput Assist Radiol Surg 7(2):305313

35. Brinkman WM, Buzink SN, Alevizos L, de Hingh IHJT, Jakimowicz JJ (2012) Criterion-based laparoscopic training reduces total training time. Surg Endosc 26(4):1095-1101 